\title{
Salivary stimulation-could it play a role in GERD management?
}

\begin{abstract}
Multiple diverse studies indicate that swallowed saliva plays an important role in neutralizing stomach acid refluxed into the esophagus and mouth; the acid that causes the symptoms of gastro-esophageal reflux disease (GERD). This article briefly reviews the epidemiology of GERD, its pathogenesis and symptom presentation, its connection to salivation, and its medical management and presents results from a study suggesting that an increase in salivation during sleep can significantly improve symptoms associated with the condition. The presented study demonstrates that an OTC product currently on the market (OraCoat XyliMelts) that is specifically designed to increase salivation through the slow release of flavoring is an effective adjunctive remedy for reducing reflux and heartburn symptoms associated with sleep related GERD.
\end{abstract}

Volume 10 Issue 3 - 2018

\author{
Jeffrey Burgess \\ Oral Care Research Associates, USA
}

Correspondence: Jeffrey Burgess, Oral Care Research Associates, 2006 NE 63rd Street Seattle, Washington 981 I5, USA, Tel 2064502640

Email oral.care.research.assoc@gmail.com

Received: February 12, 2018 | Published: May 02, 2018

\section{Overview}

GERD (gastro-esophageal reflux disease) describes a condition in which the lower esophageal sphincter (LES) relaxes and allows stomach acid to flow up into the esophagus and towards/into the mouth. Symptoms develop when reflux is excessive and the esophagus and mouth is bathed in acid for a long enough period of time to cause mucosal damage. GERD is estimated to occur in ten to thirty percent of the population in developed countries. ${ }^{1}$

Nocturnal GERD involves reflux of acid into the esophagus during sleep. The condition is estimated to occur once a month in up to $43 \%$ of individuals and once a week in $20 \%$ of the population. ${ }^{2,3}$ GERD is associated with heartburn (upper chest pain), an acid taste at the back of the throat (regurgitation or reflux) ) $^{4,5}$ as well as voice hoarseness and sleep disturbance. ${ }^{6,7}$

In the primary care setting, GERD is typically diagnosed via symptom presentation and response to empiric trials of proton pump inhibitors (PPIs). Specialized tests are only considered necessary if there is suspicion of more severe esophageal abnormality. ${ }^{8}$ Guidelines for diagnosing GERD have been published by the American College of Gastroenterology and the American Gastroenterological Association. ${ }^{9}, 10$

There are several 'defense' elements that help to mitigate GERD including the lower esophageal sphincter (LES) which keeps acid within the stomach, esophageal peristalsis which mechanically propels fluid (including saliva) into the stomach, and saliva flow which serves to neutralize and dilute acid that escapes the stomach. ${ }^{11,12}$ Prolonged contact with stomach acid can lead to mucosal damage in the esophagus and to oral structures. The physiologic abnormalities that cause GERD include poor functioning of the LES, abnormal esophageal clearance, reduced salivary production, altered esophageal mucosal resistance, and delayed gastric emptying. ${ }^{13}$

\section{Saliva and GERD}

It has been proposed that increased salivation resulting from esophageal acidification is mediated through an 'esophago-salivary' reflex. ${ }^{14}$ Acid accumulating in the upper region of the esophagus is reported to reflexively initiate saliva production. ${ }^{15}$ But this reflexive stimulation does not appear to occur as readily when stomach acid is infused into the lower esophagus and when it does not reach the upper regions of the GI track. ${ }^{16}$ Thus saliva, with its innate acid buffering capacity, appears to play a role in mediating some of the symptoms of GERD, and its affect may be more pronounced when saliva is stimulated by acid reaching the upper esophagus.

Saliva is acknowledged to play in important role in protecting the esophageal lining, partly by diluting and partly by buffering stomach acid that enters the esophagus through reflux. ${ }^{17-20}$ Swallowing occurs at the rate of about once per minute during the day under normal circumstances while moving saliva, and food, into the esophagus ${ }^{21}$ but is apparently altered by food consumption and age. ${ }^{18}$ Saliva at rest and during stimulation differs significantly. The normal resting, or unstimulated, whole salivary flow rate is $0.25-0.50 \mathrm{~mL}$ (or gram) $/ \mathrm{min}$. Symptomatic dryness may not be reliably observed until the level falls below $0.10 \mathrm{~mL}$ (or gram) $/ \mathrm{min}$. The normal, whole stimulated (by chewing paraffin) salivary flow rate is $1-3 \mathrm{~mL}$ (or gram)/min. As a general rule, $0.7 \mathrm{ml} / \mathrm{min}$ is the cut off point for defining normal versus abnormal stimulated flow of whole saliva and $0.1 \mathrm{ml} / \mathrm{min}^{22}$ is the cutoff for below-normal unstimulated whole salivary flow.

Saliva production is reduced during sleep. Age also plays a significant role in the production of saliva and, in addition, may also contribute to disturbances in esophageal motility and peristalsis as well as nonpropulsive and repetitive contractions. Saliva production is also reduced by numerous medications such as the anti-convulsants, anti-parkinsonian agents, anti-psychotics, anti-depressants, anti-pruritics, anti-histamines, anti-hypertensives, anxiolytics, expectorants, decongestants, diuretics, narcotics, monoamine oxidase inhibitors, sedatives, systemic bronchodilators, cardiac antiarrhythics, and skeletal muscle relaxants. In addition, medical conditions that common occur in the elderly, such as cerebrovascular disease, cardiovascular disease, pulmonary disease, diabetes mellitus, and Parkinson's disease ${ }^{23,24}$ are associated with reduced salivation as well as altered esophageal motility and sphincter function.

\section{Current treatment of GERD}

The medical standard of care for treating GERD is pharmacotherapy that involves prescribed and over-the-counter (OTC) medications 
which inhibit acid secretion (proton pump inhibitors: PPI's), histamine receptor antagonists ( $\mathrm{H} 2$ blockers), and prokinetics that increase tone in the lower esophageal sphincter. Alternative remedies include dietary modification, avoiding meals and alcohol shortly before sleep and raising the head of the bed, and over-the-counter (OTC) alkaline agents (e.g. calcium carbonate) that help to neutralize stomach acid.

In at least one study, PPI's and H2 blockers were found to be equivalent in terms of symptomatic relief. ${ }^{25}$ Other studies suggest greater symptomatic improvement may occur with use of the PPIs ${ }^{22}$. However, the management of GERD with PPIs may not be helpful in all cases. It has been suggested that up to one-third of GERD patients may not respond to - PPI intervention. ${ }^{27}$ PPI use has also been associated with an increased risk of bone fracture as well asVitamin B-12 deficiency. ${ }^{28}$

The use of histamine- 2 receptor antagonists ${ }^{29}$ may also be limited by their side effects and, due to their inhibition of the liver P450 system, the potential for drug interactions. ${ }^{30}$ This class of medication has not been well studied in pregnant women. Hence, the literature suggests that patients planning on becoming pregnant or who are pregnant should consult their family physician prior to their use. Caution should also be used in those individuals who have allergies to certain medicines, foods, kidney or liver problems; or certain lung diseases such as COPD, diabetes, or a history of porphyria. ${ }^{31,32}$ Medications that increase tone in the esophageal sphincter have also been recommended for use in severe cases of GERD. These prokinetic medications are only recommended for short term use and their side effect spectrum (e.g. depression and severe muscle twitching, dizziness or lightheadedness, potentially fatal heart arrhythmias) limits their general usefulness. ${ }^{33}$

Antacids are effective in managing acute GERD but are not considered reasonable for long term use ${ }^{34}$ due to their side effects. Further, for GERD occurring at night, it is not until sleep is disturbed that these medications are taken, which reduces their overall benefit in terms of sleep quality of life. ${ }^{35,36}$ Given the fact that many people do not gain relief from PPIs, that histamine-2 receptor antagonists are associated with multiple side effects and interactions with other drugs, and that the benefit of antacids is limited by associated sleep disturbance (having to repeatedly awaken to take the medication) and by the fact that they may not be appropriate for chronic use, additional approaches to the treatment of GERD occurring during sleep need to be considered..$^{37}$

\section{Study rationale}

The study was conducted by Peter Van der Ven, Michael Karcher, and myself and published in August 2017. ${ }^{38}$ The aim of this study was to see if OraCoat XyliMelts, an OTC dissolvable, adhering disc used to reduce excessive day time or night time dry mouth, would also reduce reflux and heartburn occurring during sleep. OraCoat XyliMelts are made from food grade ingredients and stimulate saliva via slowly released flavor when used as directed. Reflux symptoms are sometimes more prominent at night because acid can more readily enter the esophagus while patients are lying down. Anecdotal reports and research suggest that salivary stimulants can reduce the sensation of dry mouth. As noted, the literature suggests that salivation can ameliorate reflux and heartburn ${ }^{39}$. Hence, we hypothesized that the stimulation of salivation by a slowly dissolving flavored intraoral adhering disc could also significantly alter reflux and heartburn symptoms associated with GERD during night-time sleep.

\section{Subjects and methods}

\section{Study design}

This study was approved by the Western Institutional Review Board on September 16, 2014 (WIRB; 1019 39th Ave S, Ste 120, Puyallup, WA 98374). It was designed as a randomized, placebocontrolled trial involving two over the counter products currently on the market for use in the management of dry mouth symptoms. The product of interest was cleared by the FDA for investigation in the context of GERD on September 4, 2014 (Investigational New Drug Application number 123574 US FDA). Study information/results can be found on ClinicalTrials.gov.

Study participants and the research coordinator were blinded as to the product received by the subjects who were independently randomized. The product of interest was OraCoat XyliMelts, produced by OraHealth Corporation in Bellevue, Washington. The ingredients in OraCoat XyliMelts are all-natural and commonly used in foods: xylitol for sweetness, mild mint for additional flavor, cellulose gum to slow dissolution and lubricate the mouth, an acacia gum adhesive layer, and calcium carbonate to neutralize the acidity of acacia gum and oral bacteria. It has been reported that when the product is dissolved in 5 parts water the resulting $\mathrm{pH}$ is 8.138 . Product users also report that OraCoat XyliMelts discs slowly dissolve over several hours during sleep and their flavor can still be sensed upon awakening 8 hours later.

The product used as a placebo was a water based gel containing cellulose hydrocolloid gums with sorbitol and xylitol sweeteners. As it is a soluble gel introduced prior to sleep, it was presumed to be eliminated from the oral cavity fairly quickly via salivary stimulation. For this reason it was selected as a placebo.

\section{Study population/subjects}

Subjects were drawn from individuals living in major metropolitan cities in the United States who responded to an ad soliciting paid volunteers.

Those qualifying for the study based on inclusion and exclusion criteria were, after consent, assigned to a baseline information collecting period of two weeks. Each day subjects were required to complete a short questionnaire that included the following questions:

a. Did you taste refluxed stomach acid during your sleep last night (yes/no);

b. How severe was the reflux (mild, moderate, severe, very severe);

c. Did you have heartburn when you slept (yes/no);

d. How severe was the heartburn (mild, moderate, severe, very severe);

e. Did you keep water by your bedside because of dry mouth occurring during sleep (yes/no);

f. Did you have uncomfortable dry mouth when you slept or upon awakening (yes/no);

g. Did you experience hoarseness of your voice in the morning (yes/ no);

h. Did you need to take antacids during sleep (yes/no); and

i. If so, how many did you take (number). 
Upon completion of this first baseline phase, qualified subjects were then entered into the product phase of the study (phase two). Qualification for entry into phase two was based on having reported reflux taste on eight of the 14 days of baseline and dry mouth seven of the same mornings. Randomization was to one of two groups: treatment or control. Each subject then received by mail either the adhering discs disguised in unmarked packaging (treatment), or a sweet, water based gel in an unmarked white tube (control), with printed instructions copied from the manufacturer's recommendations for their method of use at bedtime. The analyzed variables of interest included reflux taste, reflux severity, heartburn sensation, heartburn severity, morning voice hoarseness and antacid use during sleep time. 53 subjects were ultimately selected to receive the disc or gel to use during sleep for two additional weeks. Comparisons were then made within and between groups for all outcome variables.

\section{Results}

Subjects in the two final intervention groups were not significantly different when compared on the basis of gender, age, or prior medical diagnosis of reflux. For the symptom of heartburn, Subjects using the discs demonstrated a significant reduction in reported pain of heartburn when compared with baseline (one sided U-test $\mathrm{p}$ value $<.001$ ). Subjects using the gel also experienced a significant reduction in pain when compared to baseline (U-test $p$ value $<.001$ ). When these two remedies were compared via U-test, disc intervention demonstrated significantly greater improvement in heartburn pain than gel intervention (U-test $\mathrm{p}$ value $<.01$ ) (Figure 1).

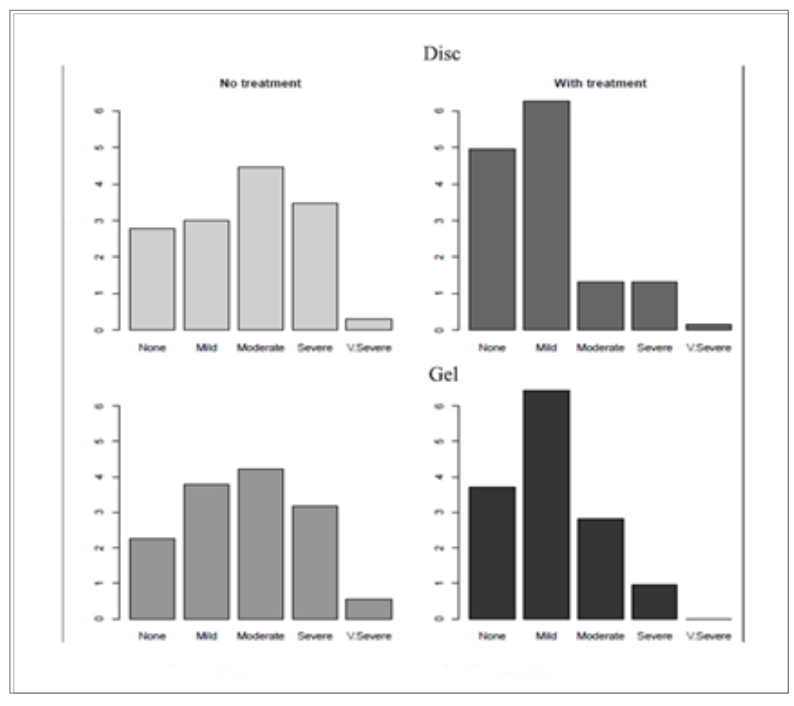

Figure I Heartburn.

In terms of reflux severity, subjects using the disc intervention reported a significant reduction in reflux severity when compared with baseline (one sided U-test p value <.001). (Figure 2) Subjects using the gel also experienced a significant reduction in reflux severity when compared to baseline values ( $U$-test $p$ value $<.001$ ). When the performance between the disc and gel treatments was compared, a statistically significant difference was not observed (U-test $\mathrm{p}>0.13$ ).

Hoarseness was also significantly reduced with disc and gel treatment $(\mathrm{p}<.001)$ with comparison between the two products not statistically significant $(\mathrm{p}=0.41)$ (Figure 3$)$. The disc treatment resulted in a $60 \%$ reduction in antacid use while the gel intervention demonstrated a $45 \%$ reduction. Values were significantly different for both treatments. A T-test comparing the two treatments did not show a significant difference $(\mathrm{p}=0.89)$ (Figure 3$)$.

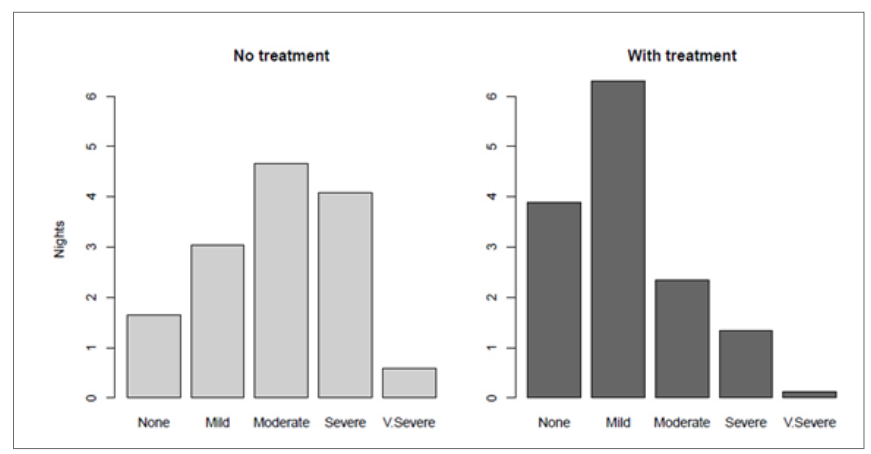

Figure 2 Reflux Severities with Disc.

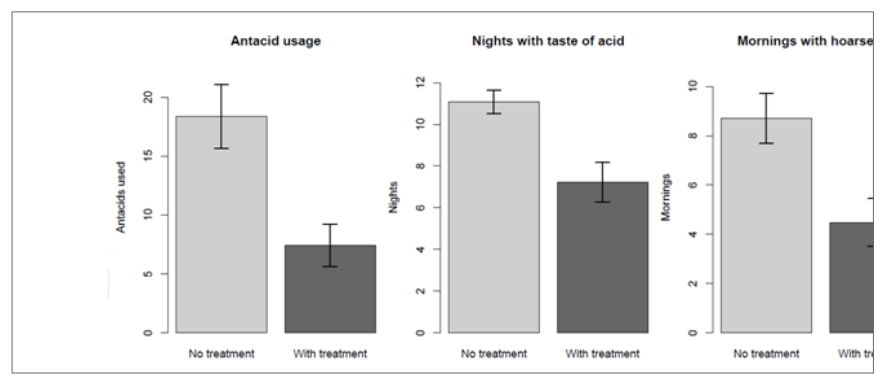

Figure 3 Antacid Uses, Nights with Taste of Acid, and Hoarseness.

\section{Discussion}

Nightly use of both adhering discs and gel dry mouth remedies that stimulate saliva via flavor appear to significantly reduced symptoms associated with GERD, including morning hoarseness, reflux acid taste, night time heartburn, and perceived reflux. Subjects who used the discs and gel for two weeks also demonstrated a significant reduction in antacid use during the night in comparison to two weeks of baseline use. The discs were found to be generally more effective in reducing symptoms than the gel, although most of the differences were not statistically significant. The exception was heartburn, where improvement was found to be significantly better for subjects using the discs than the gel. Significant side effects were not reported in either group during product use.

This reviewed study suggests that two available OTC products used to manage dry mouth during sleep may provide an effective adjunctive remedy for reducing reflux and heartburn symptoms in patients with concomitant xerostomia. The adhering discs and the gel were well tolerated and not associated with adverse reactions during use. Further, the data appear to support the hypothesis that an increase in salivation during sleep may be the reason for symptom reduction. The findings of this study are novel and medically relevant as prolonged salivary stimulation via the introduction of a persistent oral flavor during sleep may provide an additional strategy for managing symptoms arising from nocturnal GERD.

This study was limited by the lack of inclusion of different population cohorts and study duration. A prospective study involving 
different population groups (e.g. individuals with post radiation xerostomia or dryness associated with autoimmune disease) would help to further clarify the idea.

\section{Acknowledgments}

None.

\section{Conflict of interest}

The authors declare no conflict of interest.

\section{References}

1. Holtmann G. Reflux disease: the disorder of the third millennium. Eur $J$ Gastroenterol Hepatol. 2001;13(Suppl 1):S5-S11.

2. Petersen H. The prevalence of gastrooesophageal reflux disease. Scan J Gastroentero. 1995;(Suppl 211):5-6.

3. Locke GR, Talley NJ, Fett SL, et al. Prevalence and clinical spectrum of gastroesophageal reflux: a population-based study in Olmsted County, Minnesota. Gastroenterology. 1997;112(5):1448-1456.

4. Bardhan KD, Strugala V, Dettmar PW. Reflux revisited: advancing the role of pepsin. Int J Otolaryngol. 2012;2012:646901.

5. Vakil N, van Zanten SV, Kahrilas P, et al. The Montreal definition and classification of gastroesophageal reflux disease: a global evidence-based consensus. Am J Gastroenterol. 2006;101(8):1900-1920.

6. Jansson C, Nordenstedt H, Wallander MA, et al. A population-based study showing an association between gastroesophageal reflux disease and sleep problems. Clin Gastroenterol Hepatol. 2009;7(9):960-965.

7. Mody R, Bolge SC, Kannan H, et al. Effects of gastroesophageal reflux disease on sleep and outcomes. Clin Gastroenterol Hepatol. 2009;7(9):953-959.

8. Locke GR III, Talley NJ, Fett SL, et al. Prevalence and clinical spectrum of gastroesophageal reflux: a population-based study in Olmsted County, Minnesota. Gastroenterology. 1997;112(5):1448-1456.

9. DeVault KR, Castell DO. Updated guidelines for the diagnosis and treatment of gastroesophageal reflux disease. Am J Gastroenterol. 2005;100(1):190-200.

10. Kahrilas PJ, Shaheen NJ, Vaezi MF, et al. American Gastroenterological Association Medical Position Statement on the management of gastroesophageal reflux disease. Gastroenterology. 2008;135(4):13831391.

11. Kahrilas PJ. GERD pathogenesis, pathophysiology, and clinical manifestations. Cleve Clin J Med. 2003;70(Suppl 5):S4-S19.

12. Marco Patti G. Gastroesophageal Reflux Disease.

13. Kahrilas PJ, Lee TJ. Pathophysiology of gastroesophageal reflux disease. Thorac Surg Clin. 2005;15:323-333.

14. Shafik A, El-Sibai O, Shafik AA, et al. Effect of topical esophageal acidification on salivary secretion: identification of the mechanism of action. J Gastroenterol Hepatol. 2005;20(12):1935-1939.

15. Skoczylas T, Yandrapu H, Poplawski C, et al. Salivary bicarbonate as a major factor in the prevention of upper esophageal mucosal injury in gastroesophageal reflux disease. Dig Dis Sci. 2014;59(10):2411-2416.

16. Dutta SK, Agrawal K, Mahmoud MA. Modulation of salivation and heartburn in response to the site of acid infusion in the human oesophagus. Aliment Pharmacol Ther. 2010;32(6):795-800.

17. Kao CH, Ho YJ, ChangLai SP, et al. Evidence for decreased salivary function in patients with reflux esophagitis. Digestion. 1999; 60(3):191-
195.

18. Helm JF, Dodds WJ, Hogan WJ. Salivary response to esophageal acid in normal subjects and patients with reflux esophagitis. Gastroenterology. 1987;93(6):1393-1397.

19. Pope CE. Acid-reflux disorders. N Engl J Med. 1994;331(10):656-660.

20. Smith DJ, Joshipura K, Kent R, et al. Effect of age on immunoglobulin content and volume of human labial gland saliva. $J$ Dent Res .1992;71(12):1891-1894.

21. http://emedicine.medscape.com/article/2091828-overview\#aw2aab6b5

22. Hiramatsu T, Kataoka H, Osaki M, et al. Effect of aging on oral and swallowing function after meal consumption. Clin Interv Aging. 2015; 10:229-235.

23. Fox PC, Busch KA, Baum BJ. Subjective reports of xerostomia and objective measures of salivary gland performance. $J$ Am Dent Assoc. 1987;115(4):581-584.

24. Fass R, Pulliam G, Johnson C, et al. Symptom severity and oesophageal chemosensitivity to acid in older and young patients with gastrooesophageal reflux. Age Ageing. 2000;29(2):125-130.

25. Chait, MM. Gastroesophageal reflux disease: Important considerations for the older patients. World J Gastrointest Endosc. 2010;2(12): 388-396.

26. Haag S, Holtmann G. Onset of relief of symptoms of gastroesophageal reflux disease: post hoc analysis of two previously published studies comparing pantoprazole $20 \mathrm{mg}$ once daily with nizatidine or ranitidine $150 \mathrm{mg}$ twice daily. Clin Ther. 2010;32(4):678-690.

27. Armstrong D, Paré P, Pericak D, et al. Canadian Pantoprazole GERD Study Group. Symptom relief in gastroesophageal reflux disease: a randomized, controlled comparison of pantoprazole and nizatidine in a mixed patient population with erosive esophagitis or endoscopy-negative reflux disease. Am J Gastroenterol. 2001;96(10):2849-2857.

28. Vela MF. Medical Treatments of GERD: The Old and New. Gastroenterol Clin North Am. 2014;43(1):121-133.

29. Lam JR, Schneider JL, Zhao W, Corley DA. Proton pump inhibitor and histamine 2 receptor antagonist use and vitamin B12 deficiency. JAMA. 2013;310(22):2435-2442.

30. Vela MF. Medical Treatments of GERD: The Old and New. Gastroenterol Clin North Am. 2014; 43(1):121-133.

31. http://www.webmd.com/heartburn-gerd/h2-blockers-acid-reducers-forgastroesophageal-reflux-disease-gerd

32. http://livertox.nih.gov/H2ReceptorBlockers.htm

33. http://www.medicinenet.com/nizatidine-oral/page3.htm

34. http://drugs.nmihi.com/prokinetics.htm

35. Gisbert JP, Cooper A, Karagiannis D, et al. Impact of gastroesophageal reflux disease on patients' daily lives: a European observational study in the primary care setting. Health Qual Life Outcomes. 2009;7: 60.

36. Jung Hye-kyung, Choung RS, Talley NJ. Gastroesophageal Reflux Disease and Sleep Disorders: Evidence for a Causal Link and Therapeutic Implications. J Neurogastroenterol Motil. 2010;16(1):22-29.

37. http://www.mayoclinic.org/diseases-conditions/gerd/basics/treatment/ con-20025201

38. Burgess JA, Der Ven PV, Karcher MK. Effect on Acid Reflux Symptoms Occurring during Sleep of an Oral Adhering Disc Containing only Food Ingredients. J Gastrointest Dig Syst. 2017;7:524.

39. https://www.oracoat.com/pages/xylimelts-clinical-studies 